\title{
Constraining the Topology of the Universe
}

\author{
Neil J. Cornish, ${ }^{1}$ David N. Spergel, ${ }^{2}$ Glenn D. Starkman, ${ }^{3,4}$ and Eiichiro Komatsu ${ }^{2,5}$ \\ ${ }^{1}$ Department of Physics, Montana State University, Bozeman, Montana 59717, USA \\ ${ }^{2}$ Department of Astrophysical Sciences, Princeton University, Princeton, New Jersey 08544, USA \\ ${ }^{3}$ Center for Education and Research in Cosmology and Astrophysics, Department of Physics, Case Western Reserve University, \\ Cleveland, Ohio 44106-7079, USA \\ ${ }^{4}$ CERN Theory Division, CH-1211 Geneva 23, Switzerland \\ ${ }^{5}$ Department of Astronomy, The University of Texas at Austin, Austin, Texas 78712, USA \\ (Received 8 October 2003; published 19 May 2004)
}

\begin{abstract}
The first year data from the Wilkinson Microwave Anisotropy Probe are used to place stringent constraints on the topology of the Universe. We search for pairs of circles on the sky with similar temperature patterns along each circle. We restrict the search to back-to-back circle pairs, and to nearly back-to-back circle pairs, as this covers the majority of the topologies that one might hope to detect in a nearly flat universe. We do not find any matched circles with radius greater than $25^{\circ}$. For a wide class of models, the nondetection rules out the possibility that we live in a universe with topology scale smaller than $24 \mathrm{Gpc}$.
\end{abstract}

DOI: 10.1103/PhysRevLett.92.201302

PACS numbers: 98.80.Es, 98.70.Vc, 98.80.Jk

Recently, the Wilkinson microwave anisotropy probe (WMAP) has produced a high resolution, low noise map of the temperature fluctuations in the cosmic microwave background (CMB) radiation [1]. Our goal is to place constraints on the topology of the Universe by searching for matched circles in this map. Here we report the results of a directed search for the most probable topologies. The results of the full search, and additional details about our methodology, will be the subject of a subsequent publication.

The question we seek to address can be plainly stated: Is the Universe finite or infinite? Or, more precisely, what is the shape of space? Our technique for studying the shape, or topology, of the Universe is based on the simple observation that, if we live in a universe that is finite, light from a distant object will be able to reach us along more than one path. The one caveat is that the light must have sufficient time to reach us from multiple directions or, put another way, that the Universe is sufficiently small. The idea that space might be curled up in some complicated fashion has a long history. In 1900, Schwarzschild [2] considered the possibility that space may have nontrivial topology, and used the multiple image idea to place lower bounds on the size of the Universe. Recent progress is summarized in Ref. [3].

The results from the WMAP experiment [1] have deepened interest in the possibility of a finite universe. Several reported large scale anomalies are all potential signatures of a finite universe: the lack of large angle fluctuations [4], reported non-Gaussian features in the maps [5,6], and features in the power spectrum [7].

The local geometry of space constrains, but does not dictate, the topology of space. A host of astronomical observations supports the idea that space is locally homogeneous and isotropic, so we may restrict our attention to the three-dimensional spaces of constant isotropic curva- ture: Euclidean space $E^{3}$, hyperbolic space $H^{3}$, and spherical space $S^{3}$. A useful way to view nontrivial topologies with these local geometries is to imagine the space being tiled by identical copies of a fundamental cell. For example, Euclidean space can be tiled by cubes, resulting in a three-torus topology. Assuming that light has sufficient time to cross the fundamental cell, an observer would see multiple copies of a single astronomical object. To have the best chance of seeing "around the Universe" we should look for multiple images of the most distant reaches of the Universe. The last scattering surface (LSS), or decoupling surface, marks the edge of the visible Universe. Thus, looking for multiple imaging of the LSS is a powerful way to look for nontrivial topology.

But how can we tell that the essentially random pattern of hot and cold spots on the LSS have been multiply imaged? The LSS is 2-sphere centered on the observer, and each copy of the observer will come with a copy of the LSS. If the copies are separated by a distance less than the diameter of the LSS, then the copies of the LSS will intersect. Since the intersection of two 2-spheres defines a circle, the two last scattering surfaces will intersect along circles. These circles are visible by both copies of the observer, but from opposite sides. Of course, the two copies are really one observer so, if space is sufficiently small, the cosmic microwave background radiation from the LSS will have patterns of hot and cold spots that match around circles [8]. The key assumption in this analysis is that the CMB fluctuations come primarily from the LSS and are due to density and potential terms at the LSS.

Implementing our matched circle test is straightforward but computationally intensive. The general search must explore a six-dimensional parameter space. The parameters are the location of the first circle center $\left(\theta_{1}, \phi_{1}\right)$, the location of the second circle center 
$\left(\theta_{2}, \phi_{2}\right)$, the angular radius of the circle $\alpha$, and the relative phase of the two circles $\beta$. We use the HEALPIX [9] scheme to define our search grid on the sky. A resolution $r$ HEALPIX grid divides the sky into $N=12 N_{\text {side }}^{2}$ equal area pixels, where $N_{\text {side }}=2^{r}$. Similar angular resolutions were used to step through $\alpha$ and $\beta$. Thus, the total number of circles being compared scales as $\sim N^{3}$, and each comparison takes $\sim N^{1 / 2}$ operations. The simplest implementation of the search at the resolution of the WMAP data $(r=9)$ would take greater than $10^{22}$ operations. This is not computationally feasible. However, with the algorithms outlined below, we can carry out a complete search for most likely topologies using the WMAP data.

The WMAP data suggest that the Universe is very nearly spatially flat, with a density parameter $\Omega_{0}=$ $1.02 \pm 0.02$ [4]. Our universe is either Euclidean, or its radius of curvature is large compared to the radius of the LSS. For topology to be observable using our matched circle technique, we require that the distance to our nearest copy is less than the diameter of the LSS. This in turn implies that, near our location and in at least one direction, the fundamental cell is small compared to the radius of curvature. Given the observational constraint on the curvature radius, there are strong constraints on the types of spherical topologies that might be detected [10], and on where we can be located in a hyperbolic manifold for the topology to be detectable $[11,12]$. Naturally, the near flatness of the Universe places no restrictions on the observability of the Euclidean topologies. Remarkably, the largest matching circles in most of the topologies we might hope to detect will be back to back on the sky or nearly so. This immediately reduces the search space from six to four dimensions. This result holds for most circle pairs in nine of the ten Euclidean topologies (the exceptions are the largest circle pairs formed by twisted identifications, and the next-to-largest circle pairs in the Hantzsche-Wendt space). The result is less obvious in spherical space [11], but is nonetheless exact for a large class of such spaces (single action manifolds). All others (double or linked action manifolds) predict slightly less than $180^{\circ}$ separations between the circle centers for generic locations of the observer, but larger offsets for special locations. For topology to be detectable in hyperbolic manifolds, we would need to live near a "cusp" or "horn," where the topology is of the form $R \times T^{2}$ or $R^{2} \times S$ [12] and the matching circles are back to back on the sky or nearly so. The possibility of a slight offset motivates the final search described in this Letter, where we consider circles separated by between $170^{\circ}$ and $180^{\circ}$. The possibility of a less probable topology requires the full search which will be described in a future publication.

How do we compare circles? Around each pixel $i$, we draw a circle of radius $\alpha$ and linearly interpolate values at $n=2^{r+1}$ points along the circle. We then Fourier transform each circle: $T_{i}(\phi)=\sum_{m} T_{i m} \exp (i m \phi)$ and compare circle pairs, with equal weight for all angular scales:

$$
S_{i j}(\alpha, \beta)=\frac{2 \sum_{m} m T_{i m}(\alpha) T_{j m}^{*}(\alpha) e^{-i m \beta}}{\sum_{n} n\left[\left|T_{i n}(\alpha)\right|^{2}+\left|T_{j n}(\alpha)\right|^{2}\right]} .
$$

The $i, j$ label the circle centers. With this definition $S_{i j}=$ 1 for a perfect match. For two random circles, the expectation value is zero, $\left\langle S_{i j}\right\rangle=0$. We estimate $\left\langle S_{i j}^{2}\right\rangle$ below. We can speed the calculation of the $S$ statistic by rewriting it as $S_{i j}(\alpha, \beta)=\sum_{m} s_{m} e^{-i m \beta}$, where $s_{m}=$ $2 m T_{i m} T_{j m}^{*} /\left[\sum_{n} n\left(\left|T_{i n}\right|^{2}+\left|T_{j n}\right|^{2}\right)\right]$. By performing an inverse fast Fourier transform of the $s_{m}$, we get $S_{i j}(\alpha, \beta)$ at a cost of $\sim N^{1 / 2} \log N$ operations. This reduces the cost of the back-to-back search from $\sim N^{5 / 2}$ to $\sim N^{2} \log N$. We further speed the calculation by using a hierarchical approach: We first search at $r=7$ and identify the 5000 best circles at a fixed value of $\alpha$. We then refine the search around these best pixel centers and radii and complete the search at $r=9$. $S_{\max }(\alpha)$ is the best match value of $S_{i j}(\alpha, \beta)$ for all pairs of circles within a specified range of angular separations, extremized over all relative phases $\beta$.

To test the search algorithm, we generated a simulated CMB sky for a finite universe with cubic three-torus topology with side length 0.513 in units of the radius of the LSS. When only the Sachs-Wolfe effect [13] was included, the algorithm found nearly perfect circles: $S_{\max }=0.99$ with pixelization effects accounting for the residual errors. Real world effects significantly degrade the quality of the circles. The dominant sources of noise are velocities at the LSS. Following the approach outlined in the appendix of [14], we generate CMB skies with initial Gaussian random amplitudes for each $k$ mode and then compute the transfer function for each value of $k$. This approach implicitly includes all of the key physics: finite thickness LSS, reionization, the integrated Sachs-Wolfe (ISW) effect, and the Doppler term. Since topology has little effect on the power spectrum for multipoles $l>20$, we use the best fit parameters based on the analysis of the WMAP temperature and polarization data [4]. The ratio of the amplitude of the potential and density terms (which contribute to the signal for the circle statistic) to the velocity and ISW terms (which contribute to the noise for the circle statistic) depends weakly on the basic cosmological parameters: Within the range consistent with the WMAP data, the variation is small. For the simulated sky, we assume that the primordial potential fluctuations had the usual $1 / k^{3}$ power spectrum. The simulation included detector noise [15] and the effects of the WMAP beams [16]. The simulation did not include gravitational lensing of the CMB as the lensing deflection angle in the standard cosmology is small [17], $\sim 6^{\prime}$, compared to the smoothing scale used in the analysis, $40^{\prime}$. When the search was performed on these realistic skies, the quality of circle matches was degraded. Figure 1 shows the results of the search on the simulated sky: The peaks in the plots correspond to radii 


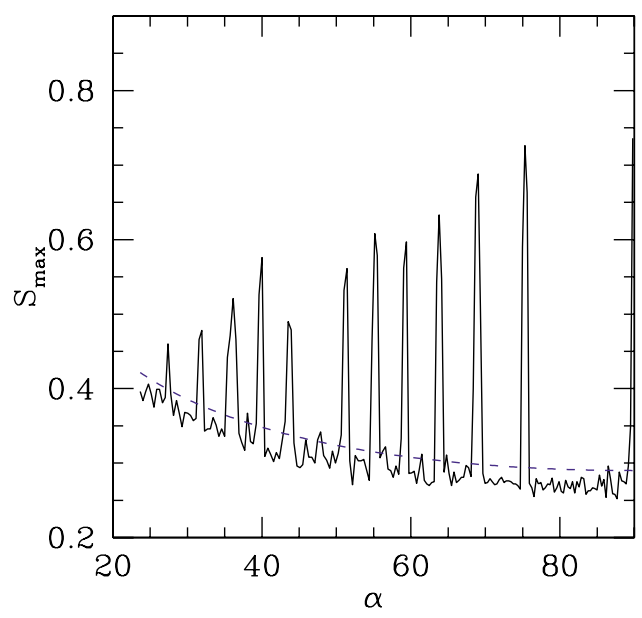

FIG. 1 (color online). The maximum value of the circle statistic as a function of radius, $\alpha$ (in degrees), for a simulated finite universe model. The peaks in the plots correspond to positions of matched circles. The dashed (blue) line corresponds to the detection threshold described below.

at which a matched circle was detected. The largest circles had $S_{\max }=0.75$, and the best match value decreased for smaller circles. The pure Doppler terms are correlated for large circles $\left(\alpha>45^{\circ}\right)$, and anticorrelated for small circles. The code was able to find all 101 predicted circle pairs with radii greater than $25^{\circ}$ : The poorest detection had an $S_{\max }$ value of 0.45 . While we only simulated a three-torus, the degradation of the circle match as a function of radius will be nearly identical for other topologies as it is determined by local physics at angular scales well below the circle radius. We will report in more detail on such tests in a future publication.

For the circle search, we generated a low foreground CMB map from the WMAP data. Outside the WMAP Kp2 cut, we used a noise-weighted combination of the WMAP $\mathrm{Q}, \mathrm{V}$, and $\mathrm{W}$ band maps. Using the template approach of [18], these maps were corrected for dust, foreground, and synchrotron emission. This map was smoothed to the resolution of the Q band map. Inside the $\mathrm{Kp} 2$ cut, we used the internal linear combination map [18].

The false positive signal can be estimated by approximating the CMB sky as a Gaussian random field with coherence angle $\theta_{c} \sim 0.7^{\circ}$. There are roughly $N_{\text {circ }}=2 \pi / \theta_{c}^{2}$ independent circles on the sky of radius $\alpha$. Along each circle, there are $2 \pi \sin (\alpha) / \theta_{c}$ independent patches and also $2 \pi \sin (\alpha) / \theta_{c}$ independent orientations. Thus, the back-to-back search considered $N_{\text {search }}=4 \pi^{2} \sin (\alpha) / \theta_{c}^{3}$ possible circle pairs. If we treat the patches as independent, then $\left\langle S^{2}\right\rangle^{1 / 2}=$ $1 / \sqrt{2 \pi \sin (\alpha) / \theta_{c}}$. The number of circles expected above a given threshold $S_{0}$ is $N\left(S>S_{0}\right)=$ $N_{\text {search }} / 2 \operatorname{erfc}\left(S_{0} / \sqrt{2\left\langle S^{2}\right\rangle}\right)$. Thus, inverting the complementary error function yields the maximum value for any false positive circle at each radius:

$$
S_{\max }^{\text {f.p. }}(\alpha) \simeq\left\langle S^{2}\right\rangle^{1 / 2} \sqrt{2 \ln \left(\frac{N_{\text {search }}}{2 \sqrt{\pi \ln \left(N_{\text {search }}\right)}}\right)} .
$$

This estimate is sensitive to $\theta_{c}$, and excludes correlations between circles.

We use "scrambled" versions of the WMAP cleaned sky map to obtain a more accurate estimate of the false positive rate. We generate scrambled versions of the true sky by taking the spherical harmonic transform of the map and then randomly exchanging $a_{l m}$ values at fixed $l$. This scrambling generates new maps with the same twopoint function but different phase correlations. The $S_{\max }$ value for the best fit back-to-back circle found at each radius is plotted as a function of $\alpha$ in Fig. 2. The false positive signal is well fit by a slightly modified form of the analytical estimate (2). Based on the analytical estimate, we set a detection threshold so that fewer than 1 in 100 random skies should generate a false match. This threshold is shown in Figs. 1-3.

Figure 3 shows the result of the back-to-back search on the WMAP data. The search checked circle pairs that were both matched in phase (points along both circle labeled in a clockwise direction) and flipped in phase. These two cases correspond to searches for nonorientable and orientable topologies. We did not detect any statistically significant circle matches in either search. The simulations show that the minimum signal expected for a circle of radius greater than $25^{\circ}$ is $S_{\max }=0.45$, which is above the false detection threshold. Since we did not detect this signal, we can exclude any universe that predicts circles larger than this critical size. In a broad class of topologies, this constrains the minimum

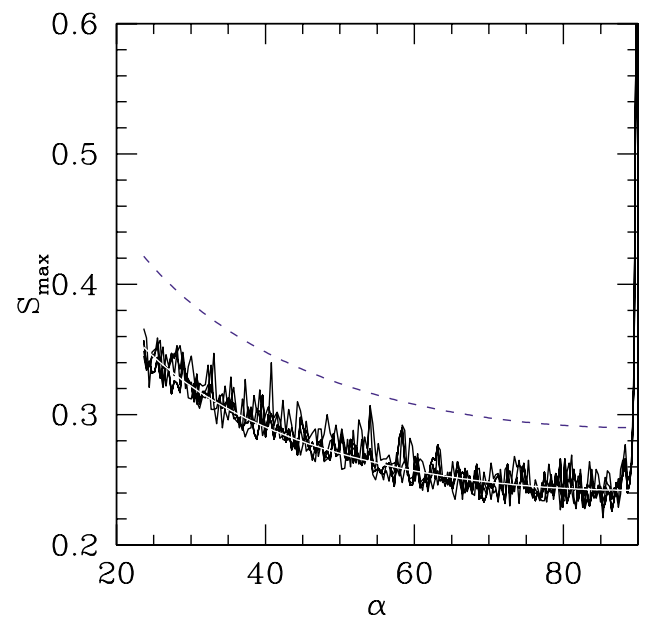

FIG. 2 (color online). The maximum value of the circle statistic as a function of radius for back-to-back circle pairs on scrambled CMB skies. The results are shown for seven different realizations. The white line is a fit for the false positive rate. The dashed (blue) line shows the false detection threshold set so that fewer than 1 in 100 simulations yield a false event. 


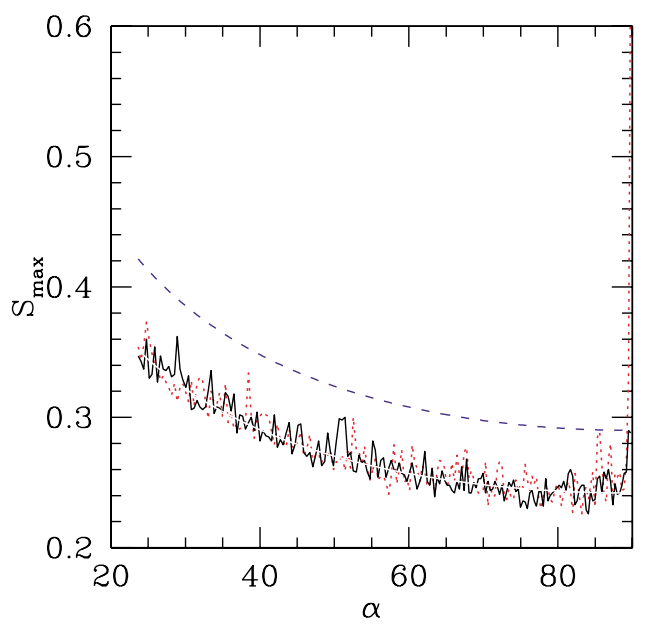

FIG. 3 (color online). The maximum value of the circle statistic as a function of radius for the WMAP data for backto-back circles. The dotted (red) line in the figure is for an orientable topology. The solid line is for nonoriented topologies. The white line is the expected false detection level. The dashed (blue) line is the detection threshold. The spike at $90^{\circ}$ is due to a trivial match between a circle centered at one point and its copy centered around the antipodal point.

translation distance $d=2 R_{c} \operatorname{atan}\left[\tan \left(R_{l s s} / R_{c}\right) \cos \left(\alpha_{\min }\right)\right]$, where $R_{c}$ is the radius of curvature, and $R_{l s s}$ is the distance to the last scattering surface. Note that one recovers the Euclidean formula for $R_{c} \rightarrow \infty$ and the hyperbolic formula for $R_{c} \rightarrow i R_{c}$. For a flat or nearly flat universe, models with $d<2 R_{l s s} \cos \left(25^{\circ}\right) \simeq 24 \mathrm{Gpc}$ are excluded. Note that the search excludes the Poincare dodecahedron suggested in [7] as this model predicts back-to-back circles of radius $35^{\circ}$.

By considering a broader set of circles, we can extend the search to other possible topologies. Figure 4 shows the

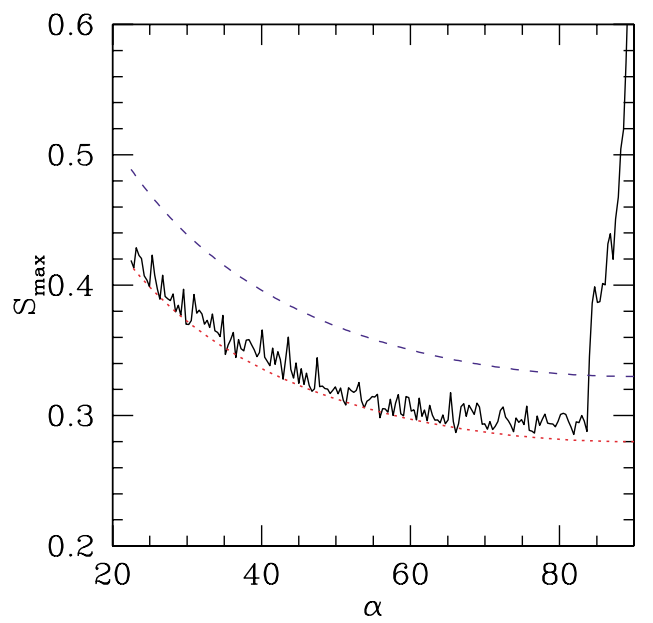

FIG. 4 (color online). The maximum value of the circle statistic as a function of radius for the WMAP data for circles whose centers are separated by greater than $170^{\circ}$. The solid line in the figure is for an orientable topology. The dotted (red) line is the expected false detection level and the dashed (blue) line is the detection threshold. result of a search for nearly back-to-back circles: circles whose centers are separated by more than $170^{\circ}$. The restricted search requires searching far more circles than the back-to-back search, which increases the false positive signal. This increases the minimum detectable angular radius to $28^{\circ}$. The signal at $\alpha \sim 85^{\circ}-90^{\circ}$ is due to nearly overlapping and osculating circles. This feature is also seen in scrambled skies.

Has this search ruled out the possibility that we live in a finite universe? No, it has only ruled out a broad class of finite universe models smaller than a characteristic size. By extending the search to all possible orientations, we will be able to exclude all topologies out to $\sim 24 \mathrm{Gpc}$. More directed searches can extend the result for specific manifolds somewhat beyond $24 \mathrm{Gpc}$. With lower noise and higher resolution CMB maps (from the extended mission of WMAP and from Planck), we will be able to search for smaller circles and extend the limit to $\sim 28 \mathrm{Gpc}$. If the universe is larger than this, the circle statistic will not be able to constrain its shape.

We thank the WMAP team for their wonderful sky maps and for insightful comments on the draft. We thank the LAMBDA data center for providing the data for the analysis. We acknowledge the use of the following software packages: HEALPIX, CMBFAST, and Waterloo MAPLE. G. D. S. is supported by a DOE grant at CWRU.

[1] C. L. Bennett et al., Astrophys. J. Suppl. 148, 1 (2003).

[2] K. Schwarzschild, Vier Ast. Ges. 35, 337 (1900); J. M. Stewart and M. E. Stewart, Classical Quantum Gravity 15, 2539 (1998), English translation.

[3] J. J. Levin, Phys. Rep. 365, 251 (2002).

[4] D. N. Spergel et al., Astrophys. J. Suppl. 148, 175 (2003).

[5] A. de Oliveria-Costa, M. Tegmark, M. Zaldarriaga, and A. Hamilton, Phys. Rev. D 69, 063516 (2004).

[6] H. K. Eriksen, F. K. Hansen, A. J. Banday, K. M. Gorski, and P. B. Lilje, astro-ph/0307507.

[7] J.-P. Luminet, J. R. Weeks, A. Riazuelo, R. Lehoucq, and J.-P. Uzan, Nature (London) 425, 593 (2003).

[8] N. J. Cornish, D. N. Spergel, and G. D. Starkman, Classical Quantum Gravity 15, 2657 (1998).

[9] K. M. Gorski, E. Hivon, and B. D. Wandelt, http://www. eso.org/science/healpix/

[10] J. R. Weeks, R. Lehoucq, and J.-P. Uzan, Classical Quantum Gravity 20, 1529 (2003).

[11] J. R. Weeks, Mod. Phys. Lett. A 18, 2099 (2003).

[12] B. Mota, G. I. Gomero, M. J. Reboucas, and R. Tavakol, astro-ph/0309371.

[13] R. K. Sachs and A. M. Wolfe, Astrophys. J. 147, 738 (1967).

[14] E. Komatsu et al., Astrophys. J. Suppl. 148, 119 (2003).

[15] G. Hinshaw et al., Astrophys. J. Suppl. 148, 63 (2003).

[16] L. Page et al., Astrophys. J. Suppl. 148, 39 (2003).

[17] U. Seljak, Astrophys. J. 463, 1 (1996).

[18] C. L. Bennett et al., Astrophys. J. Suppl. 148, 97 (2003). 